\title{
Prácticas Pedagógicas en el Proceso de Transición hacia la Escuela Inclusiva. Seis Experiencias en El Salvador
}

\author{
Pedagogical Practices in the Transition Process towards an \\ Inclusive School. Six Experiences in El Salvador
}

\author{
Cristina Aracely Muñoz Morán * \\ Universidad Evangélica de El Salvador, El Salvador
}

\begin{abstract}
El presente artículo analiza seis prácticas pedagógicas desarrolladas en centros educativos salvadoreños durante el año 2016, considerando aspectos como la cultura escolar, la participación de actores sociales y la contextualización de los aprendizajes. La investigación es cualitativa, a partir del diseño de estudio de casos y contó con la participación de directores, docentes y madres de familia. Entre los hallazgos obtenidos se destaca la vinculación que generalmente establecen los docentes con los estudiantes, pues se basa en el reconocimiento y aceptación de la persona desde su diferencia. Y dicha vinculación es la que ha favorecido la participación de las familias y la comunidad en el proceso educativo e ir transformando poco a poco las prácticas pedagógicas, pues difícilmente la escuela se vuelve más inclusiva sino se pone la mirada en el encuentro con el otro.
\end{abstract}

Descriptores: Práctica pedagógica; Participación de los padres; Participación estudiantil; Aprendizaje; El Salvador.

\begin{abstract}
This article analyzes six pedagogical practices developed in Salvadoran schools during 2016, considering aspects such as school culture, the participation of social actors and the contextualization of learning. The research is qualitative, based on the design of case studies and with the participation of school principals, teachers and mothers. Among the findings obtained is the bonding that teachers usually establish with students, since it is based on the acknowledging and acceptance of the person based on their difference. And this connection is what has favored the participation of families and the community in the educational process and is gradually transforming pedagogical practices, since it will be difficult for schools to become more inclusive unless they focus on encountering one another.
\end{abstract}

Keywords: Teaching practice; Parent participation; Student participation; Learning; El Salvador.

\section{Introducción}

Lograr que la educación sea un derecho fundamental para la niñez y la adolescencia es un compromiso adquirido por El Salvador a través de la firma de diferentes documentos (elaborados por la Organización de las Naciones Unidas para la Educación y Diversificación, la Ciencia y la Cultura: Declaración Mundial sobre la Educación para Todos en Jomtien (1990), Declaración de Salamanca (1994) y la Declaración de Lima (2014), entre otros), el cual también se refleja en el proceso de transformación gradual en el que se encuentra inmerso el sistema educativo salvadoreño a fin de consolidar la educación inclusiva. Dicho sistema se organiza en cuatro niveles educativos: parvularia,

\begin{tabular}{|c|c|c|}
\hline *Contacto: cristina.munoz@uees.edu.sv & $\begin{array}{l}\text { Recibido: } \\
1^{\text {a }} \text { Evaluación: }\end{array}$ & $\begin{array}{l}14 / 11 / 2017 \\
20 / 01 / 2018\end{array}$ \\
\hline ISSN: $0718-7378$ & Aceptado: & $26 / 02 / 2018$ \\
\hline
\end{tabular}


básica, media y superior, y dos modalidades: educación de adultos y educación especial (Ministerio de Educación, 1994).

Por tanto, a la fecha en el país continúan existiendo escuelas de educación especial con el objetivo de brindar "una atención educativa específica permanente o transitoria a la población estudiantil en condición de discapacidad intelectual, dando respuesta a sus características y necesidades individuales" (Ministerio de Educación, 2016d, p. 10). Además, estas escuelas tratan de gestionar los apoyos y las condiciones que favorezcan el desarrollo de aprendizajes académicos y funcionales para que los estudiantes logren su plena participación en la comunidad.

También se valora la influencia que tiene la comunidad y la cultura en la que está inmersa la escuela en el proceso de consolidación de la educación inclusiva, pues en el hecho educativo participan diferentes actores. $\mathrm{Y}$ en este escenario las prácticas pedagógicas se convierten en uno de los ejes estratégicos para eliminar las barreras para la participación y el aprendizaje de los estudiantes, entendidas como los obstáculos que estos pueden experimentar durante su proceso educativo y que pueden surgir "a partir de la interacción de los estudiantes en sus contextos, su comunidad y las realidades particulares de cada centro" (Ministerio de Educación, 2010, p. 8).

Por tanto, siendo las practicas pedagógicas un elemento clave para dinamizar la inclusión (Ávila y Esquivel, 2009), éstas contribuirán a que la escuela se articule con la familia y la comunidad (Comellas, 2013; Finocchio y Legarralde, 2007), para garantizar la contextualización de los aprendizajes que se proponen a los estudiantes (Arnaiz y Azorín, 2013; Baldares, 1998). Por ello, durante el proceso de investigación se recopilaron seis experiencias de buenas prácticas en educación inclusiva (Organización de Estados Iberoamericanos, 2014) de centros educativos públicos con el objetivo de visibilizar los avances alcanzados en el proceso de transición hacia una escuela más inclusiva.

Ahora bien, cabe mencionar que en el contexto salvadoreño se puede observar que ocurre la paradoja de las paradojas que comentan Echeita y Domínguez (2011), pues desde las escuelas de educación especial se están generando cambios en la forma de valorar y relacionarse con los estudiantes con discapacidad. Pues no sólo es que los estudiantes se sienten estimados en dichas escuelas, sino que también los docentes están promoviendo estrategias ${ }^{1}$ que contribuyan a sensibilizar a las familias y a la comunidad sobre el derecho a la educación y a la participación de estos.

Entonces, el análisis de las experiencias de dichos centros es relevante, pues hasta la fecha (2018) no hay documentos publicados que muestren el proceso de transición hacia una escuela inclusiva a partir de las prácticas pedagógicas, por lo que este primer acercamiento puede generar nuevas líneas de investigación y debate que orienten las futuras propuestas en el tema.

\footnotetext{
${ }^{1}$ Las escuelas promueven la inclusión de los estudiantes en centros educativos regulares y les dan seguimiento (Ministerio de Educación, 2016d), participan en los Comités Departamentales de Apoyo a la Inclusión o apoyan a otras escuelas por medio de procesos de formación docente en temas relacionados con la atención a la diversidad.
} 


\section{Revisión de la literatura}

\subsection{La educación inclusiva en El Salvador}

El sistema educativo salvadoreño ha definido como una prioridad la transformación educativa, desde un modelo constructivista, humanista y socialmente comprometido. Por ello, el Plan Social Educativo 2009-2014: "Vamos a la escuela", propone la educación inclusiva como la ruta que guíe el cambio, pues "brinda oportunidades equitativas de aprendizaje a niños, niñas y jóvenes independientemente de sus diferencias sociales, culturales y de género, así como de sus diferencias en las destrezas y capacidades" (Ministerio de Educación, 2009a, p. 87). Por tanto, la inclusión es una invitación a ir al encuentro con el otro, en una relación basada en la igualdad en tanto sujetos de derecho y en el respeto a la diferencia. Y para ello, se crea la Política de Educación Inclusiva (Ministerio de Educación, 2009b) con el objetivo de garantizar que el sistema educativo se centre en la persona, considerando su diversidad personal, cultural y ecológico-social (Ávila y Esquivel, 2009).

En este contexto, se valora la participación de diferentes actores en la gestión de la educación inclusiva, pues se considera que a partir de la articulación de los esfuerzos de dichas personas se logrará generar un espacio que permita el intercambio de experiencias y el desarrollo de buenas prácticas, lo cual facilitará la trasformación gradual de la educación para responder a las demandas de una cultura inclusiva (Ministerio de Educación, 2009b). Por ende, se puede visualizar que la inclusión conlleva una transformación de la educación desde sus cimientos, afectando los diferentes niveles educativos y a los actores vinculados con el proceso de aprendizaje.

Además, en el país se están realizando acciones que favorezcan la transición de enfoques homogéneos a enfoques que consideren la diversidad con igualdad (Blanco y Duk, 2011), al menos en lo que se refiere a desarrollar sistemas de apoyo que complementen la labor de los docentes, pues se ha creado los siguientes:

- Estrategia del Docente de Apoyo a la inclusión, la cual apoya a los docentes del aula regular para atender a la población estudiantil, en especial a aquellos que "presentan barreras para el aprendizaje y la participación, mediante la identificación de sus dificultades de aprendizaje, situaciones de discapacidad, niñez trabajadora, o con capacidades o talentos excepcionales, estudiantes con sobre edad y otros" (Ministerio de Educación, 2014a).

- Comités Departamentales de Apoyo a la Inclusión, quienes son los responsables de articular a los diferentes actores educativos que se encuentren el territorio a fin de "dinamizar en el departamento los procesos de inclusión educativa de los estudiantes identificados en riesgo de exclusión, garantizando el acceso, permanencia de calidad y egreso efectivo del sistema educativo" (Ministerio de Educación, 2016b).

- Centros de Orientación y Recursos son un "servicio de apoyo del sistema educativo que brinda atención psicopedagógica a la población estudiantil en riesgo de exclusión, tanto en las escuelas de educación especial como en los centros educativos regulares" (Ministerio de Educación, 2016a).

Por otra parte, aún está pendiente la flexibilización y diversificación de la oferta educativa, pues únicamente se cuenta con la enseñanza en escuelas regulares o de la modalidad de 
educación especial, tanto en el ámbito público como en el privado. Además, no se cuenta con currículos accesibles que den respuesta a todos los estudiantes, se utilizan los mismos programas de estudio en todas las escuelas, pero en la modalidad de educación especial solo se desarrollan los programas de educación inicial, parvularia, y primero y segundo grado con sus respectivas adecuaciones curriculares (Ministerio de Educación, 2016c).

En lo que se refiere al desarrollo profesional docente, en los últimos años se revisaron los planes de estudio de la formación inicial docente, donde se concluyó que:

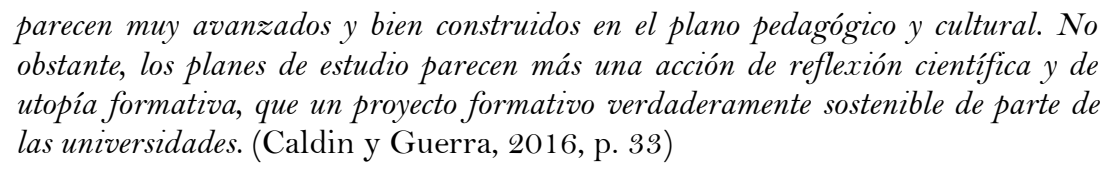

Mientras que en el Plan nacional de formación de docentes en servicio en el sector público (Ministerio de Educación, 2014b) se incluyó como una de las áreas de especialización, el apoyo al desarrollo de la educación inclusiva. Si bien ha habido avances en cuanto la formación docente, aún falta darle a la educación inclusiva un rol protagónico que permee los diferentes niveles del sistema educativo, en todas sus dimensiones.

\subsection{Desarrollo de buenas prácticas en educación inclusiva}

Las prácticas pedagógicas son el eje vertebrador desde el cual se dinamizan los aprendizajes y a través de estas se vinculan docentes, estudiantes, familia y comunidad; por ello, tienen gran relevancia en el contexto escolar. De acuerdo con la Política de Educación Inclusiva (Ministerio de Educación, 2009b), las prácticas de gestión pedagógica tienen el objetivo de "desarrollar una gestión que propicie prácticas inclusivas en el centro educativo y en el aula (p. 29).” Por tanto, al visualizar las prácticas pedagógicas más allá del aula, como tradicionalmente han sido entendidas, se encuentra el reto de diseñarlas desde el enfoque de inclusión, tomando en cuenta aspectos tales como: las características de los estudiantes y de su entorno, las relaciones que se han establecido entre escuelafamilia-comunidad, el valor que la sociedad le asigna a la educación o el grado de participación que tienen los actores educativos en la toma de decisiones referidas al proceso educativo, entre otros.

Entonces, las prácticas pedagógicas no se pueden comprender solamente como una ejecución de propuestas de aprendizaje, sino como un proceso dinámico que surge de la interacción de personas diferentes y que pretende dar respuesta a una realidad sociocultural heterogénea en un espacio y tiempo determinado (Arnaiz, 2000). Esto conlleva a desarrollar una visión sistémica de las prácticas pedagógicas, lo que implicaría una mayor participación de estudiantes, familia y comunidad local en la educación por medio de la creación de espacios de discusión y toma de decisiones que garanticen el derecho a una educación de calidad para todos y todas.

Por tanto, habrá acciones educativas que acerquen más que otras a consolidar la educación inclusiva. Al respecto, la Organización de Estados Iberoamericanos (2014), afirma que no hay buenas prácticas ideales, sino que dependen del contexto en el que se desarrollan. Por tanto, la práctica adquiere sentido en una realidad concreta, pues se desarrolla en unas condiciones socioculturales e individuales propias que son su referente.

De esta manera, la concepción de práctica pedagógica se amplia, más allá del quehacer educativo del docente en el aula, pues implica diferentes niveles de actuación, tanto a nivel escolar como comunitario. Además, dichos espacios de participación de la familia y la 
comunidad favorecerían a que estos gradualmente se conviertan en tomadores de decisión sobre la agenda educativa, reconociendo así el valor social de la educación como bien común.

\subsection{Generando una cultura inclusiva para la participación}

La educación inclusiva supone una actitud y un compromiso con la tarea de contribuir a una educación de calidad, por ello es importante que la escuela abra espacios para la participación de los diversos actores sociales que están inmersos en la acción educativa, pues implica una responsabilidad recíproca (Echeita y Domínguez, 2011). Además, la inclusión educativa implica desarrollar "lazos de pertenencia a una totalidad cultural, a un tejido social, sin ignorar los puntos de partida, las diferencias y la diversidad de los contextos culturales en los que habitan y construyen su identidad" (Finocchio y Legarralde, 2007, p. 10).

De esta manera, se reconoce que la inclusión supone la producción de vínculos pedagógicos significativos desde el lugar particular que cada actor ocupa en la escuela, y que dichos vínculos son influenciados por la cultura de la comunidad en donde se desarrollan. Así, la cultura espontáneamente puede tener apertura a la inclusión de la diversidad o generar exclusión al etiquetar la diferencia. En este último escenario, es preciso reconocer en el otro a un igual a partir del diálogo y la convivencia, y estar en la disposición de afectar al otro y dejarse afectar por este, para lograr una auténtica relación de encuentro con el otro (Skliar, 2008).

Por otra parte, el encuentro con el otro permite tanto el desarrollo colectivo como el crecimiento personal, pues es en la convivencia donde se da la oportunidad de generar relaciones basadas en lo diverso y lo igual, donde cada uno construye su propia idea del otro desde su historia, a fin de reconstruir la trama social que aglutina a los individuos (Comellas, 2013; Finocchio y Legarralde, 2007).

Ahora bien, la escuela tiene una función clave como parte del sistema social, pues puede generar acciones que promuevan el cambio, integrando los esfuerzos con la familia y la comunidad para desarrollar una cultura inclusiva, basada en el respeto a la persona y en la equidad. Por tanto, la escuela está llamada a promover la inclusión en el territorio, para comenzar a generar cambio en las prácticas, creencias, culturas y relaciones entre los ciudadanos (Ministerio de Educación, 2009b).

Finalmente, no podemos creer que la mera presencia física de diversos estudiantes en las aulas logre por sí misma producir inclusión y menos aún inclusión educativa (Finocchio y Legarralde, 2007). Es necesario, generar un cambio de actitud, una nueva manera de estar juntos, de convivir desde el respeto y valoración de la diferencia; estableciendo relaciones horizontales y democráticas para construir juntos una mejor educación para todos y todas.

\subsection{Contextualización de los aprendizajes desde la persona y su entorno}

El contexto va más allá de la geografía, es un espacio de construcción cultural creado por los aportes de diversas iniciativas individuales, sociales, educativas y políticas. Esto conlleva, a tener una visión sistémica de la educación, pues el contexto donde se desarrollan los aprendizajes es el punto de partida para diseñar e implementar las propuestas educativas. Por ende, el currículo que se desarrolle en el centro educativo debe caracterizarse por ser abierto y flexible, para poder incluir a todos los estudiantes, así como su contexto familiar y social. Además, la UNESCO (2004) afirma que "el currículum 
abarca todas las experiencias de aprendizaje disponibles para los estudiantes en sus escuelas, así como en sus comunidades" (p. 104).

Por tanto, la incorporación del contexto en el desarrollo del currículo es un reto para los docentes, pues es una invitación a cambiar la mirada, a ver más allá del aula, pues el aprendizaje se ve influenciado tanto por el lugar y el tiempo donde se desarrolla como por los actores que participan en su construcción. Ahora bien, si la escuela no logra abrirse hacia la comunidad, estaría convirtiéndose en una isla, volviendo a cometer el error de la educación tradicional, donde se desligaba el proceso de aprendizaje de su contexto y de su valor social. Entonces, contextualizar los aprendizajes implica considerar las condiciones en las que estos se desarrollan, para poder adecuarlos y hacerlos significativos de acuerdo con las necesidades y características de los estudiantes (Baldares, 1998).

También a través del currículo formal de las escuelas se deben planificar las situaciones de aprendizaje que se implementarán a nivel de aula, sin olvidar que también existen experiencias potenciales de aprendizaje que son difíciles de planificar, tales como las interacciones entre los estudiantes, las interacciones entre estudiantes y maestros y las situaciones de aprendizaje que se dan en la familia y en la comunidad (Ávila y Esquivel, 2009). En esta línea, Arnaiz y Azorín (2013) afirman que un currículo flexible trata de ser una respuesta a las características e intereses de los estudiantes, quienes se encuentran en contextos educativos diversos.

Por otra parte, en el proceso de planificación y evaluación de los aprendizajes también es necesario considerar la adecuación curricular, pues son los ajustes que se realizan para brindarle a cada estudiante lo que cada uno requiere para lograr los objetivos de aprendizaje del nivel o los sugeridos en la propuesta curricular, tomando en cuenta las diferencias personales, sociales o culturales (Esquivel, 2009). Además, realizar las adecuaciones curriculares implica tomar decisiones en el proceso de planificación a partir de la evaluación del estudiante y su contexto, con el fin de dar respuesta a las necesidades individuales y del grupo clase. Difícilmente se desarrollará un currículo que dé respuesta al estudiante y a su contexto, sino se parte del reconocimiento de este como persona.

\section{Método}

Para el estudio se optó por un diseño de investigación cualitativo, a fin de comprender e interpretar cómo se está consolidando la educación inclusiva en seis centros educativos públicos, a través de las prácticas que estos implementan. Por tanto, se recogieron de manera descriptiva los aportes de docentes, directoras y directores y madres de familia de las escuelas seleccionadas. Dichos aportes fueron interpretados desde las categorías de análisis definidas para la presente investigación: práctica docente inclusiva, cultura escolar, participación de actores sociales y contextualización de los aprendizajes. Así, el estudio tuvo como objetivo conocer cómo se desarrollan las prácticas pedagógicas desde el enfoque de educación inclusiva en los seis centros educativos seleccionados.

También en la investigación se utilizó la metodología del estudio de casos múltiples (Hernández Sampieri, Fernández-Collado y Baptista, 2006), para abordar cada una de las realidades en las que se desarrollan las prácticas docentes, considerando: las características del entorno en cual están inmersas, los actores que participan en la construcción de los aprendizajes y las relaciones que se establecen entre escuela y familia.

Las técnicas que se utilizaron en la investigación para recoger la información fueron: 
- Lista de cotejo se aplicó con el objetivo de tener una imagen inicial de la escuela en dos grandes áreas: datos generales y experiencias en educación inclusiva a partir de las dimensiones de culturas inclusivas y prácticas inclusivas².

- Entrevistas semi-estructuradas abordando las dimensiones: prácticas pedagógicas, cultura escolar, participación de actores y la planificación de los aprendizajes desde el enfoque de inclusión. Dichas entrevistas fueron grabadas.

- Sistematización de experiencias, la cual se realizó a partir de la reflexión y discusión colectiva de cada equipo docente, brindándoles un instrumento que recogía tanto la descripción de la experiencia como su evaluación.

Y la población que participó en la investigación estaba conformada por 23 docentes, 5 directores y 5 madres de familia de los centros educativos seleccionados, los cuales dieron su consentimiento para participar en la investigación y divulgar los resultados obtenidos. Las escuelas que se incluyeron en el estudio de casos múltiples fueron dos centros escolares regulares y cuatro escuelas de la modalidad de educación especial, ubicadas en diferentes departamentos del país: San Salvador, La Libertad, Sonsonate, La Paz y Cuscatlán. Se seleccionaron dichas escuelas a partir de los siguientes criterios: antecedentes de experiencias de buenas prácticas pedagógicas, participación en procesos de formación docente, procesos de inclusión de estudiantes de la escuela de educación especial en centros educativos regulares e inclusión de personas con discapacidad en dichos centros, ubicación geográfica del centro educativo (zona urbana o rural), escuela de educación especial y centro educativo regular.

Para el procesamiento y análisis de la información se utilizaron matrices de congruencia basadas en las cuatro categorías de análisis establecidas, en dichas matrices se vació la información obtenida teniendo en cuenta el instrumento y la fuente de información (docente, director, madre de familia). A partir de las matrices se analizaron los datos y se clasificaron los aportes obtenidos en función de los siguientes criterios: relevancia y relación con lñas categorías de análisis definidas, nivel de acuerdo o desacuerdo entre las opiniones de los diferentes participantes y otros hallazgos. Finalmente, la información recolectada en cada uno de los instrumentos utilizados también se contrastó con los aportes teóricos sobre la práctica docente desde el enfoque de inclusión.

\section{Resultados}

En este apartado se describirán los resultados obtenidos a través del proceso de recolección de información, de acuerdo con los objetivos de investigación propuestos. Cada apartado contiene los principales hallazgos encontrados.

\subsection{Desarrollo de las prácticas pedagógicas desde el enfoque de educación inclusiva}

La trasformación de la escuela por medio de la implementación de prácticas pedagógicas inclusivas ha sido una de las líneas de acción propuestas por el Ministerio de Educación, y para ello es importante que docentes, directores y familias conozcan qué es inclusión y lo que implica. Así, los diferentes actores de las seis escuelas del estudio coinciden en que la educación inclusiva es para todos, es un derecho que implica ampliar la atención

${ }^{2}$ Para estas dimensiones se seleccionaron algunos de los indicadores propuestos en el instrumento de Adecuación del índice de inclusión al contexto educativo salvadoreño (Ministerio de Educación, 2010). 
educativa para brindar igualdad de oportunidades en la escuela. A continuación, se presentan algunos de los aportes al respecto:

La educación inclusiva es que nosotros como maestros y como institución debemos tomar en cuenta a todos los niños para darles una atención integral en el grado que ellos se encuentran. (Docente, centro escolar regular)

Me arriesgo a decir que el lema que se maneja aqui es todos somos iguales y cuando dicen que todos somos iguales sería incorporar a nuestros alumnos a las escuelas normales, entonces es revolucionario. (Madre de familia, escuela de educación especial)

En general, docentes y familias presentan una visión favorable de la educación inclusiva, que implica garantizar el acceso a la escuela y la incorporación de los estudiantes a la sociedad. Ahora bien, no hay consenso entre directores y docentes sobre las barreras en el aprendizaje, pues no todos opinan que dichas barreras se pueden originar debido a las políticas, el currículo o los enfoques de enseñanza que se utilizan.

Por otra parte, las madres de familia siguen visualizando la inclusión sólo hacia las personas con discapacidad, como comentaba una madre "yo siento que la educación inclusiva es para los niños con necesidades especiales porque si ellos necesitan más apoyo que un niño digamos normal" (Madre, escuela de educación especial). Mientras que directores y docentes visualizan la educación inclusiva de una manera más amplia, como afirman "la educación inclusiva es que todos los alumnos tienen derecho a la educación, no dejar a ningún alumno afuera” (Directora, centro escolar regular).

Además, la mayor parte de directores y docentes coinciden en que casi siempre la inclusión es un proceso que debe reflejarse en la planificación curricular que realiza el docente a partir de las experiencias previas de los estudiantes, como comenta una docente:

\section{La planificación es desde el aula, pero también institucional porque nosotros le hacemos llegar a la directora todo, cómo planificamos, cómo evaluamos y antes del inicio de año, nosotros nos ponemos de acuerdo cómo lo vamos a hacer. (Docente, escuela de educación especial)}

También, docentes, directores y madres valoran de forma similar varios aspectos de la práctica pedagógica que se desarrolla en la escuela, y generalmente en los puntos en que docentes y directores tienen diferente opinión, se observó que los directores tienden a tener una opinión más favorable sobre dichos puntos, como se muestra en el cuadro 1.

Además, durante la investigación se recogieron seis experiencias de prácticas pedagógicas, cada una refleja una parte del proceso de trasformación gradual por el que están pasando las escuelas y algunas de las estrategias propuestas por el MINED para dinamizar el cambio, como los Centros de Orientación y Recursos ${ }^{3}$; y los Docentes de Apoyo a la Inclusión. Cabe destacar la experiencia del Centro de Orientación y Recursos ubicado en la escuela de educación especial de Cuscatlán, el cual además de brindar atención psicopedagógica a los estudiantes y sus familias, apoya a las instituciones educativas del territorio por medio de talleres de formación, procesos de sensibilización a docentes, elaboración de material pedagógico, entre otros.

Cuadro 1. Síntesis de las opiniones de docentes y directores sobre las prácticas pedagógicas, puntos de acuerdo y desacuerdo

${ }^{3}$ Los Centros de Orientación y Recursos están conformados por un equipo multidisciplinar actualmente están funcionando 16 centros en todo el país (Ministerio de Educación, 2016a). 


\begin{tabular}{|c|c|}
\hline $\begin{array}{c}\text { ACUERDOS ENTRE DIRECTORES Y } \\
\text { DOCENTES }\end{array}$ & $\begin{array}{c}\text { DIFERENCIA DE OPINIONES ENTRE DIRECTORES Y } \\
\text { DOCENTES }\end{array}$ \\
\hline $\begin{array}{l}\text { La inclusión es un proceso que } \\
\text { implica la participación del } \\
\text { estudiante y su familia. } \\
\text { Docentes realizan la planificación } \\
\text { curricular a partir de las } \\
\text { experiencias previas de los } \\
\text { estudiantes. }\end{array}$ & $\begin{array}{l}\text { El tipo y espacios de participación en la escuela de los } \\
\text { estudiantes durante el proceso educativo. } \\
\text { Percepción de la evaluación de los aprendizajes de los } \\
\text { estudiantes, motivación que se le brinda al personal } \\
\text { para desarrollar y compartir conocimientos. } \\
\text { El aprendizaje de los docentes a partir de las } \\
\text { experiencias de colegas de otros centros educativos }\end{array}$ \\
\hline
\end{tabular}

Nota: Información obtenida por medio de las listas de cotejo aplicadas a los docentes y directores de los seis centros educativos del estudio.

Fuente: Elaboración propia.

Por otra parte, una de las experiencias vividas en el centro escolar del departamento de La Libertad, la Docente de Apoyo a la Inclusión inició su labor cambiando la percepción que tenían los estudiantes del aula de apoyo, convirtiéndola en un espacio abierto donde todos podían asistir y escoger un libro, un material educativo o un juego; donde también los docentes pueden acudir en busca de apoyo y orientación para enriquecer su práctica, como comenta la directora:

Con el Docente de Apoyo a la Inclusión, ya los alumnos no van a estar ahí en la educación especial, sino que van a estar en el aula regular y esto les ayuda también a los niños para su autoestima, que no estén aislados, y de esto se trata la inclusión. (Directora, centro escolar regular)

De esta manera, se valora que la educación inclusiva supone el establecimiento de vínculos pedagógicos significativos desde los cuales se dinamice el currículo. Así, el equipo docente de la escuela de educación especial del departamento de San Salvador realizó el proceso de planificación y secuenciación de los aprendizajes que se desarrollarían en los diferentes ciclos educativos ${ }^{4}$, estableciendo los contenidos que se abordaran en cada ciclo a partir de los programas de estudio prescritos para esta modalidad educativa.

Y en este esfuerzo de dar respuesta a las condiciones específicas de la población estudiantil y de su contexto, el equipo docente de una de las escuelas de educación especial del departamento de Sonsonate implementó una Guía de habilidades adaptativas para contribuir al buen desempeño en la vida cotidiana de los estudiantes con discapacidad intelectual. Mientras que, en la otra escuela de educación especial de Sonsonate se desarrolló el proyecto del aula hogar ${ }^{5}$, que implicó tanto la construcción de la infraestructura del aula como las propuestas para desarrollar habilidades de la vida diaria.

Por otra parte, se destaca la experiencia del centro escolar del departamento de La Paz, ubicado en una zona rural y que a partir del año 2011 incluyó a los estudiantes en situación de discapacidad en las aulas, pues antes se atendían en el Aula de Educación Especial' ${ }^{6}$. La comunidad educativa de dicha escuela ha pasado por un proceso de cambio actitudinal, transformando las percepciones erróneas que tenían acerca de la situación de discapacidad,

\footnotetext{
${ }^{4}$ Las escuelas de educación especial se organizan en ciclo educativos desde la Educación Parvularia a la Formación Prelaboral, las cuales retoman los programas de estudio de los niveles de Educación Parvularia, y de primero y segundo grado de Educación Básica.

${ }^{5} \mathrm{El}$ aula hogar es una casa construida adentro de la escuela, que tiene todas las dependencias de una casa y que se utiliza para desarrollar las habilidades para la vida de manera práctica.

${ }^{6}$ El aula de Educación Especial era un espacio donde se brindaba atención educativa a estudiantes con discapacidad en los centros escolares regulares de manera segregada, estas aulas han ido desapareciendo gradualmente para hacer efectiva la educación inclusiva en el país.
} 
para valorar a la persona y sus capacidades, lo cual ha influido en la forma de relacionarse entre estudiantes, estudiantes-docentes, docentes-familias, etc.; como comenta una docente:

Hay estereotipos que nos son tan fáciles de romper, pero yo siento que la parte de la fase de la socialización, la aceptación, la concientización, tanto de nosotros, porque empezamos por ahi, en aceptar nosotros y ver las cosas de otra manera... he visto los cambios de como los niños antes veían a los niños con capacidades especiales y... yo siento que ahora todo normal y hay apoyo para los niños, hay más interacción y también entre nosotros. (Docente, centro escolar regular)

A través de las experiencias de las seis escuelas se comienzan a observar algunos cambios en la práctica docente actual respecto a la tradicional. Ahora bien, dichos esbozos de una nueva práctica aún coexisten cotidianamente con situaciones de falta de apertura de los docentes ante las sugerencias de los estudiantes, continúan comparando el desempeño de los estudiantes con una norma preestablecida y no se introducen cambios en la planificación en base a los resultados de la evaluación de los aprendizajes.

\subsection{Influencia de la cultura escolar en la participación de la familia y la comunidad en el proceso educativo}

En el escenario de la educación inclusiva, se valora a la escuela como un espacio productor de vínculos entre los miembros de la comunidad, y las experiencias que se dan en este contexto están empapadas de la cultura escolar. Así, en las seis escuelas estudiadas, la cultura escolar se caracterizaba, tanto por el respeto a las personas que conformaban la comunidad educativa como por su apertura a la participación de estas en las acciones de mejora educativa.

Ahora bien, el aprender juntos implica un cambio de actitud para respetar y valorar las diferencias, tanto para las familias como para los docentes, como menciona una madre:

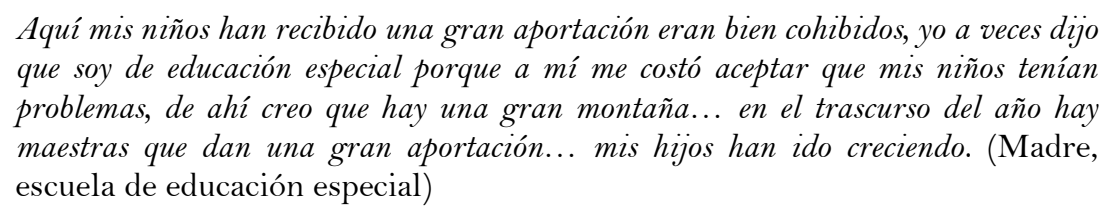

También directores y docentes manifiestan que generalmente se logra un buen clima institucional, basado en el respeto y en el esfuerzo conjunto por la mejora de los aprendizajes que se les brindan a los estudiantes; como afirman algunos docentes:

\footnotetext{
Siempre hay situaciones en las que diferimos, pero siempre llegamos a entendimiento, siempre llegamos al objetivo que es la educación de los niños. Todos estamos hacia eso, nuestra fortaleza es eso, somos unidos y buscamos la manera de salir adelante. (Docente, centro escolar regular)

Todos colaboramos, los maestros, los alumnos, los padres, todos trabajamos en conjunto. (Docente, escuela de educación especial)
}

De esta manera, los docentes valoran las relaciones basadas en el respeto y la cooperación, tanto entre docentes como docente-estudiantes y docente-familias, pues dichas relaciones sirven de modelo a los estudiantes, como se afirma a una docente "que los niños nos vean en una relación cordial y respetuosa, con lo mismo ellos aprenderán a respetarse así con sus compañeros" (Docente, escuela de educación especial). Cabe mencionar, que directores y docentes presentan acuerdos y desacuerdos en aspectos que se refieren a la cultura escolar y la participación, como se muestra en el cuadro 2. 
Cuadro 2. Síntesis de los puntos de acuerdo y desacuerdo entre docentes y directores en lo que se refiere a la cultura escolar y participación

\begin{tabular}{|c|c|}
\hline $\begin{array}{c}\text { ACUERDOS ENTRE DIRECTORES Y } \\
\text { DOCENTES }\end{array}$ & $\begin{array}{l}\text { DIFERENCIA DE OPINIONES ENTRE DIRECTORES Y } \\
\text { DOCENTES }\end{array}$ \\
\hline $\begin{array}{l}\text { La escuela y la comunidad tienen un } \\
\text { ambiente acogedor e inclusivo para } \\
\text { toda la niñez, incluyendo los } \\
\text { estudiantes con discapacidad. }\end{array}$ & $\begin{array}{l}\text { Desarrollo de actividades que fomenten la empatía, } \\
\text { cooperación y aprendizaje entre pares. } \\
\text { El estudiantado y las familias comprenden que se } \\
\text { pueden esperar diferentes logros de aprendizaje } \\
\text { según sus características. }\end{array}$ \\
\hline $\begin{array}{l}\text { Se valora a los estudiantes por ellos } \\
\text { mismos, como personas, y no en } \\
\text { relación con su rendimiento o } \\
\text { notas. }\end{array}$ & $\begin{array}{l}\text { El trabajo en equipo y el respeto entre el personal } \\
\text { docente es un modelo para estudiantes y comunidad. } \\
\text { Solicitar la opinión de los estudiantes sobre cómo se } \\
\text { podría mejorar la escuela. }\end{array}$ \\
\hline $\begin{array}{l}\text { Casi siempre se ofrece a las familias } \\
\text { una variedad de oportunidades } \\
\text { para involucrarse en la escuela. }\end{array}$ & $\begin{array}{l}\text { Se muestra a las familias formas de apoyar el trabajo } \\
\text { escolar de sus hijos en casa, y se les permite } \\
\text { involucrarse en la toma de decisiones en la escuela. } \\
\text { Involucramiento de la escuela en actividades de la } \\
\text { comunidad y viceversa. }\end{array}$ \\
\hline
\end{tabular}

Nota: Información obtenida por medio de las listas de cotejo aplicadas a los docentes y directores de los seis centros educativos del estudio.

Fuente: Elaboración propia.

Entonces, la cultura escolar va más allá de los valores, creencias y experiencias compartidas; pues implica una forma de convivir con el otro a partir de dichos elementos; por lo que es importante tenerlos en cuenta para generar las condiciones que promuevan la inclusión y la participación de la familia y la comunidad en el proceso educativo. Por tanto, se hace necesario que en la escuela se generen espacios de toma de decisiones colectivos para definir la educación que se necesita en el contexto donde se encuentra inmersa. Al respecto, algunos docentes comentan que las familias son tomadas en cuenta al elaborar el Proyecto Educativo Institucional:
Ahí participamos los docentes, la asamblea de padres también, porque se les hacen a ellos las propuestas de las actividades que vamos a realizar y son ellos quienes las aprueban, nuestra directora y también se incluyen a los estudiantes. (Docente, centro escolar regular)
En reuniones de padres de familia pedimos que nos den lineamientos... generalmente hago las siguientes preguntas ¿̇cómo queremos ver la escuela?, ¿qué es lo que nos gusta? rentonces, yo procuro que ellos expresen su sentir, y sus expectativas, de eso retomamos y se incluye. (Director, escuela de educación especial)

Sin embargo, cabe mencionar que algunas escuelas también incorporan a representantes de la comunidad en la elaboración del proyecto educativo, como comenta la docente de una de las escuelas de educación especial de Sonsonate "todas y todos, todas las docentes y todos los padres de familia, los que pertenecen al CDE [Consejo Directivo Escolar] y a veces algunos de la comunidad porque hay personas de la comunidad que nos colaboran".

Además, se puede observar que hay mayor conciencia de directores y docentes sobre la importancia de la participación de la familia y la comunidad en la educación, pero ya en acciones concretas las opiniones difieren. Ahora bien, habría que valorar el alcance de dicha participación estudiantes-familia-comunidad, para evitar que no sea una acción meramente simbólica, sino que realmente tenga poder de cambio. Y más allá de dichos espacios a nivel institucional se cuenta con el aula, donde se desarrolla el proceso educativo, al respecto los docentes manifiestan que valoran los aportes de las familias a dicho proceso: 
Tenemos reuniones de grados y las entregas de notas son en una como casa abierta, en las que el padre viene, retira las notas y tiene el espacio también de hacer interrogantes con el docente del grado. (Docente, centro escolar regular)

El padre de familia también colabora en hacer material didáctico o en preparar a su hijo para que él exponga o para que él traiga algo, participe con algo. (Directora, escuela de educación especial)

Finalmente, las seis experiencias de las escuelas reflejan que es necesario partir de la valoración de la persona y de una cultura inclusiva para promover la participación de los diferentes actores en el hecho educativo, reconociendo el aporte de cada uno.

\title{
3.3. Planificación y evaluación de los aprendizajes a partir del contexto
}

Diseñar situaciones de aprendizaje que sean significativas para los estudiantes implica partir de la realidad personal y social de estos, como comenta una docente "hay contenidos que nosotros no podemos realizarlos como vienen en el programa, entonces lo que hacemos es hacerlos según nuestro entorno... entonces, siempre hay adecuaciones de los contenidos, de acuerdo a lo qué es nuestro entorno" (docente, centro escolar regular). Por tanto, frecuentemente los docentes utilizan la comunidad con un recurso del apoyo para el proceso de aprendizaje y a las familias como facilitadoras de recursos. Así, algunos docentes tratan de incorporar a las familias con un rol más activo en el proceso educativo de sus hijos, como se menciona a continuación:

\begin{abstract}
Nosotros tenemos madres tutoras, que en el caso de seño Cecy que están un poco más avanzados, en veces ella tiene una cita médica y le dice yo voy a ir de tal hora a tal hora al seguro, pero ella deja su madre tutora y ella regresa acá, ya sabe que ella le va a desarrollar su clase o lo que le ha dejado para los alumnos. (Docente, escuela de educación especial)
\end{abstract}

Por tanto, poco a poco la escuela está involucrando a las familias y a la comunidad en el proceso educativo, y docentes y directores coinciden en que es necesario tomar en cuenta las diferencias culturales y del contexto para establecer las normas de comportamiento y seleccionar los materiales curriculares que se utilizarán. Además, docentes y directores presentan diferentes opiniones cuando se refiere a la utilización de los espacios y recursos de la comunidad como parte del proceso de enseñanza-aprendizaje, o el apoyo de las familias y otros miembros de la comunidad son utilizados en el aula; los cuales se presentan en el cuadro 3.

Cuadro 3. Síntesis de las opiniones de docentes y directores sobre la contextualización de los aprendizajes

\begin{tabular}{lc}
\hline ACUERDOS ENTRE DIRECTORES Y DOCENTES & $\begin{array}{c}\text { DIFERENCIA DE OPINIONES ENTRE } \\
\text { DIRECTORES Y DOCENTES }\end{array}$ \\
\hline $\begin{array}{l}\text { Los materiales curriculares reflejan el contexto } \\
\text { sociocultural y las experiencias de todos los } \\
\text { estudiantes. }\end{array}$ & $\begin{array}{c}\text { Los espacios y recursos de la comunidad son } \\
\text { incluidos como parte fundamental del } \\
\text { proceso de enseñanza-aprendizaje. }\end{array}$ \\
$\begin{array}{l}\text { Los deberes de la casa se relacionan con las } \\
\text { capacidades y conocimientos de los }\end{array}$ & $\begin{array}{c}\text { Las familias y otros miembros de la } \\
\text { estudiantes. }\end{array}$ \\
$\begin{array}{l}\text { Se tienen en cuenta las diferencias culturales y } \\
\text { del contexto cuando se establecen las normas } \\
\text { de comportamiento }\end{array}$ & $\begin{array}{l}\text { apoyo en las aulas. } \\
\text { Nota: Información obtenida por medio de las listas de cotejo aplicadas a los docentes y directores }\end{array}$ \\
de los seis centros educativos del estudio. & \\
Fuente: Elaboración propia.
\end{tabular}


Por otra parte, la contextualización de los aprendizajes también es un elemento común en las seis experiencias, pues en cada escuela se elaboró una propuesta para responder a una necesidad determinada, ya sea la adecuación curricular y secuenciación de los aprendizajes a nivel institucional, la elaboración de programas para desarrollar habilidades adaptativas o apoyando los procesos de inclusión, por medio de la labor del Docente de Apoyo a la Inclusión y de los Centros de Orientación y Recursos (figura 1).

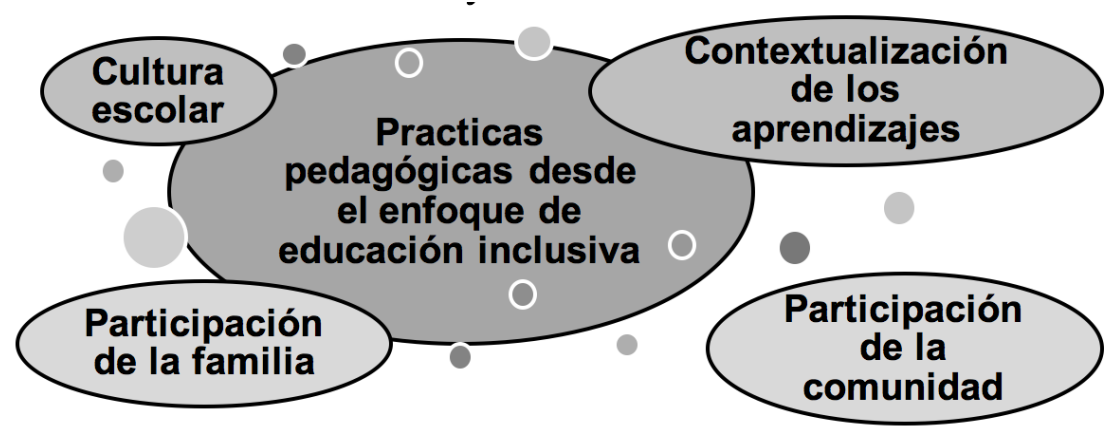

Figura 1. Relación de las categorías de análisis ${ }^{7}$ a partir de los resultados obtenidos en el proceso de investigación

Fuente: Elaboración propia.

Ahora bien, como se observa en la figura 1 la participación de la familia y especialmente de la comunidad aún se encuentra en proceso. En el caso de la familia, se han abierto algunos espacios para que pueda involucrarse en el proceso educativos de sus hijos e hijas; pero aún los directores y docentes se resisten a compartir el proceso educativo como coeducadores, incluso para realizar el seguimiento de los avances de sus hijos(as) en el hogar. Mientras que la participación de la comunidad en el discurso de docentes y directores se valora como un elemento positivo, ya en práctica queda reducida a aportes específicos y puntuales, generalmente para satisfacer las necesidades materiales de la escuela. Aún los docentes no visualizan a las personas y espacios de la comunidad como recursos de apoyo para el aprendizaje.

\section{Conclusiones}

En las experiencias de los seis centros escolares un elemento común es la valoración de la persona que hacen directores y docentes, y al reconocer las características personales y del contexto de los estudiantes, tratan de incluirlas en el proceso de aprendizaje. Así, a partir de dicha valoración han generado las diferentes prácticas pedagógicas, ya sea que estén referidas a la planificación curricular, a la vinculación con la comunidad o al desarrollo de programas de habilidades para la vida. Por otra parte, también se destaca la influencia de la cultura escolar en el desarrollo de las prácticas inclusivas, pues ha sido un aspecto clave en el proceso de sensibilización de estudiantes, familias y docentes; el cual ha facilitado el desarrollo de una convivencia basada en el respeto y valoración de la diferencia.

\footnotetext{
${ }^{7}$ Práctica docente inclusiva, participación de actores sociales familia-comunidad, cultura escolar y contex tualización de los aprendizajes.
} 
También se observaron otros elementos que han dinamizado el desarrollo de prácticas inclusivas en las seis escuelas estudiadas, tales como el liderazgo participativo de los directores, el trabajo colaborativo que se desarrolla entre docentes, y la contextualización de los aprendizajes a partir del estudiante y su entorno.

Si bien, de momento la inclusión está más clara en el discurso de directores, docentes y familias que en la práctica; es necesario visibilizar los esfuerzos que se están realizando en los seis centros escolares para que sigan proponiendo acciones que favorezcan la consolidación de la educación inclusiva. En este contexto, es importante destacar que en varias ocasiones directores y docentes no son conscientes de los cambios que están desarrollando en la escuela, por lo que necesitan que desde afuera se les reconozca.

Por otra parte, la existencia de los Centros de Orientación y Recursos aún es desconocida para muchos docentes, y en el caso de los Docentes de Apoyo a la Inclusión, no se tiene claridad de las funciones que tienen asignadas, lo cual obstaculiza el proceso de implementación y aceptación de la estrategia.

Por tanto, algunos de los retos que las seis escuelas comparten para consolidar la educación inclusiva son:

- Valorar que la inclusión educativa no sólo se refiere a las personas con discapacidad, sino a cualquier estudiante en riesgo de exclusión.

- Incluir de una forma más activa a la familia y a la comunidad en el desarrollo del proceso de educativo (planificación-ejecución-evaluación).

- Involucrar a los estudiantes en la evaluación de su aprendizaje y el de sus compañeros, y garantizar que los resultados obtenidos sirvan como insumo para generar propuestas de mejora.

- Promover el trabajo en equipo y la cooperación entre docentes, docentes-familia, docentes-estudiantes.

Finalmente, la educación inclusiva supone una actitud y un compromiso con la tarea de contribuir a una educación equitativa y de calidad. Por tanto, es importante que las escuelas abran más espacios para la participación de los diversos actores sociales que están inmersos en el hecho educativo. Ya que, en las experiencias de las seis escuelas, se observó que la participación de las familias y la comunidad se limita a acciones que no están directamente relacionadas con el proceso de aprendizaje.

\section{Recomendaciones}

A partir de los resultados obtenidos durante la investigación se proponen las siguientes recomendaciones a los directores de los seis centros escolares y funcionarios del MINED responsables de la atención y seguimiento técnico-pedagógico a dichas escuelas:

- Motivar a los docentes a que reflexionen sobre su práctica y que la sistematicen, pues generalmente no son conscientes de los esfuerzos que realizan cotidianamente para mejorar la calidad de los aprendizajes. Por tanto, se hace necesario que creen espacios para valorar los logros alcanzados, así como para aprender de las experiencias vividas.

- Generar espacios de intercambio de buenas prácticas pedagógicas entre docentes de diferentes escuelas, para fortalecer el desarrollo profesional de estos y 
fomentar el trabajo colaborativo. Dichos espacios pueden ser conversatorios, ferias de logros, tour docentes, entre otros.

- Promover la creación de más espacios de participación en los centros escolares, que impliquen a la familia y a la comunidad de forma activa, compartiendo la toma de decisiones y el diseño de propuestas para la mejora de la escuela.

- Articular las estrategias propuestas por el MINED, Centros de Orientación y Recursos y Docente de Apoyo a la Inclusión, con la práctica pedagógica de los docentes de los centros escolares para potenciar los esfuerzos que se realizan en el terminar para reducir las barreras para el aprendizaje y la participación de la niñez en riesgo de exclusión. Para ello es necesario que las estrategias mencionadas sean conocidas y se vinculen con las organizaciones o instituciones del territorio.

- Ampliar el análisis del aporte que realizan los diferentes actores sociales (estudiantes, docentes, directores, familia, sociedad civil y Estado), para la consolidación de la educación inclusiva, a través de futuras investigaciones.

Finalmente, es necesario reconocer y agradecer el compromiso de los directores, docentes y madres de familia por transformar la educación, pues a través de sus experiencias, esfuerzos y dedicación están contribuyendo a construir una escuela más inclusiva y abierta a la comunidad.

\section{Referencias}

Arnaiz, P. (2000). Educar en y para la diversidad. En P. Arnaiz (Comp.), Actas del I congreso internacional de nuevas tecnología y necesidades educativas especiales: Nuevas tecnologías, viejas esperanzas (pp. 29-37). Madrid: Consejería de Educación y Universidades.

Arnaiz, P. y Azorín, C. (2013). Una experiencia de innovación en educación primaria: Medidas de atención a la diversidad y diseño universal del aprendizaje. Tendencias Pedagógicas, 22, 9-30.

Ávila, A. y Esquivel, V. (2009). Educación inclusiva en nuestras aulas. San José: Coordinación Educativa y Cultural Centroamericana.

Baldares, T. (1998). La adecuación curricular: Una estrategia para lograr la pertinencia, la equidad y la calidad de la educación. San José: SIMED-UNESCO.

Blanco, R. y Duk, C. (2011). Educación inclusiva en América Latina y el Caribe. Revista Aula, 17, $37-55$.

Caldin, R. y Guerra, L. (2016). Revisión con enfoque inclusivo de los planes de estudio de profesorado en El Salvador. San Salvador: Ministerio de Educación.

Comellas, M. J. (2013). Familia, escuela y comunidad: Un encuentro necesario. Barcelona: Octaedro.

Echeita, G. y Domínguez, A. (2011). Educación inclusiva. Argumento, camino y encrucijadas. Revista Aula, 17, 35-35.

Esquivel, V. (2009). Llevando a la práctica las adecuaciones curriculares. San José: Coordinación Educativa y Cultural Centroamericana.

Finocchio, S. y Legarralde, M. (2007). Pedagogía de la inclusión. Gestión pedagógica para equipos directivos. Caracas: Federación Internacional Fe y Alegría.

Hernández Sampieri, R., Fernández-Collado, C. y Baptista, P. (2006). Metodología de la investigación. Ciudad de México: McGraw-Hill. 
Ministerio de Educación. (1994). Fundamentos curriculares de la educación nacional. San Salvador: Ministerio de Educación.

Ministerio de Educación. (2009a). Plan social educativo 2009-2014: Vamos a la escuela. San Salvador: Ministerio de Educación.

Ministerio de Educación. (2009b). Política de educación inclusiva. San Salvador: Ministerio de Educación.

Ministerio de Educación. (2010). Adecuación del índice de inclusión al contexto educativo salvadoreño. San Salvador: Ministerio de Educación.

Ministerio de Educación. (2014a). Acuerdo No 15-0458. Lineamiento para la implementación de la estrategia educativa docente de apoyo a la inclusión. San Salvador: Ministerio de Educación.

Ministerio de Educación. (2014b). Plan nacional de formación de docentes en servicio en el sector público. San Salvador: Ministerio de Educación.

Ministerio de Educación. (2016a). Centro de orientación y recursos desde el enfoque de inclusión. San San Salvador: Ministerio de Educación.

Ministerio de Educación. (2016b). Comités departamentales de apoyo a la inclusión. San Salvador: Ministerio de Educación.

Ministerio de Educación. (2016c). Fundamentos curriculares de la educación especial. San Salvador: Ministerio de Educación.

Ministerio de Educación. (2016d). Orientaciones técnicas y administrativas de las escuelas de educación especial. San Salvador: Ministerio de Educación.

Organización de Estados Iberoamericanos. (2014). Guía para la reflexión y valoración de prácticas inclusivas. Santo domingo: OEI.

Organización de las Naciones Unidas para la Educación, la Ciencia y la Cultura. (2004). Temario abierto sobre educación inclusiva. Materiales de apoyo para responsables de politicas educativas. Santiago de Chile: UNESCO.

Skliar, C. (2008). ¿Incluir las diferencias? Sobre un problema mal planteado y una realidad insoportable. Orientación y Sociedad, 8, 1-16.

\section{Breve CV de la autora}

\section{Cristina Aracely Muñoz Morán}

Maestra en Política y Evaluación Educativa (Universidad Centroamericana José Simeón Cañas), Licenciada en Psicología (Universidad de Deusto), y Profesora en Educación Especial para el nivel básico (Universidad Católica de Occidente), es Catedrática de la Universidad Evangélica de El Salvador en el Departamento de Educación Especial. Su labor de investigación está relacionada con el desarrollo de la educación inclusiva a través de las prácticas pedagógicas, la participación de actores sociales y la gestión escolar, teniendo como punto de referencia el reconocimiento de la persona en el contexto educativo. ORCID ID: 0000-0002-1785-4884. Email: cristina.munoz@uees.edu.sv 